\title{
Relación Entre el Clima Organizacional y la Satisfacción Laboral en una Pequeña Empresa del Sector Privado
}

\section{RELATION BETWEEN ORGANIZATIONAL CLIMATE AND JOB SATISFACTION IN A SMALL ENTERPRISE FROM PRIVATE SECTOR}

\section{Walter L. Arias Gallegos', Gabriela Arias Cáceres²}

1. Psicólogo y candidato a doctor en psicología por la Universidad Nacional de San Agustín de Arequipa. Diplomado en Gestión de Recursos Humanos, Gerencia y Supervisión en Seguridad Integral y Medio Ambiente y Gerencia de Sistemas Integrados. Profesor auxiliar y docente investigador de la Universidad Católica San Pablo.

2. Administrador de negocios por la Universidad Católica San Pablo.

\section{RESUMEN}

Este estudio pretende valorar las relaciones entre el clima organizacional y la satisfacción laboral. Para ello se trabajó con un diseño correlacional y una muestra de 45 trabajadores de una pequeña empresa privada y se aplicó el Perfil Organizacional de Liker y la Escala de Satisfacción en el Trabajo de Warr, Cook y Wall. Los resultados indican que existen relaciones moderadas no significativas entre las variables, pero entre las dimensiones de flexibilidad y reconocimiento existen relaciones más fuertes y significativas con la satisfacción laboral. Además, se encontraron diferencias significativas entre los varones y las mujeres, entre el grado de instrucción y el área de trabajo del personal evaluado. Se concluye, por tanto, que el clima organizacional se relaciona con la satisfacción laboral de manera moderada.

(Arias W, Arias G, 2014. Relación entre el clima organizacional y la satisfacción laboral en una pequeña empresa del sector privado. Cienc Trab. Sep-Dic; 16 [51]: 185-191).

Palabras clave: CLIMA ORGANIZACIONAL, SATISFACCIÓN LABORAL, PSICOLOGÍA ORGANIZACIONAL.

\section{ABSTRACT}

This study pretends to value relations between organizational climate and job satisfaction. We work with a sample of 45 workers from a small private enterprise and we use Likert's Organizational Profile and the Scale of Job Satisfaction of Warr, Cook and Wall. Results indicate that there are moderate relations between our variables of study, but the dimensions of organizational climate such as flexibility and acknowledgement there are stronger correlations with job satisfaction. Moreover, we found significant differences among male and female workers, according to instruction level and the area of work in personnel who were evaluated. The conclusion is that organizational climate is related with job satisfaction moderately.

Key words: ORGANIZATIONAL CLIMATE, JOB SATISFACTION, ORGANIZATIONAL PSYCHOLOGY.

\section{INTRODUCCIÓN}

El clima organizacional y la satisfacción laboral han sido, quizá, las variables más estudiadas en el ámbito laboral. En cuanto al clima organizacional, el constructo proviene de la psicología lewiniana y su enfoque de campo ya desde 1939. ${ }^{1}$ Aunque sería Gellerman el que introduce el concepto en el ámbito organizacional en la década del 60'.

Son diversas las definiciones de clima organizacional, pero tres han sido los enfoques que han servido para conceptualizarlo. Los enfoques objetivos se concentran en las características de la orga-

\author{
Correspondencia / Correspondence: \\ Walter L. Arias Gallegos \\ Universidad Católica San Pablo \\ Urb. Campiña Paisajista s/n Quinta Vivanco, Cercado, Arequipa, Perú \\ Tel.: (51-54) 608020 \\ e-mail: walterlizandro@hotmail.com \\ Recibido: 06 de Agosto 2014 / Aceptado: 28 de Noviembre 2014
}

nización, mientras que los enfoques subjetivos se enfocan en los trabajadores. Sin embargo, los enfoques integradores toman en cuenta ambos factores. Por nuestra parte, definimos el clima organizacional como el conjunto de percepciones que tienen las personas de su centro laboral. ${ }^{2}$

En ese sentido el clima organizacional es un buen descriptor de la estructura de una organización. ${ }^{1}$ De ahí que Renis Likert plantea su modelo teórico según el cual el comportamiento de los trabajadores es causado por las características administrativas y organizacionales, así como por la información que los trabajadores poseen de la empresa, sus percepciones y capacidades. Ello supone diferenciar el clima organizacional como causa y como efecto, ya que la conducta del trabajador puede ser influida por el clima organizacional, pero también puede influir en él. ${ }^{3}$

La teoría de Renis Likert, de corte integrador, cuenta con evidencia tanto para los factores objetivos como para los factores subjetivos, ya que, por un lado, hay estudios que señalan que la estructura de la organización ${ }^{4}$ y la jerarquía del puesto ${ }^{5}$ tienen gran influencia en el clima laboral; y, por otro lado, varios estudios también reportan que el clima organizacional tiene influencia en la comunicación y las relaciones interpersonales, el compromiso del trabajador, la motivación, la salud y la seguridad en el trabajo. ${ }^{6}$ 
En un estudio que realizamos el año pasado, encontramos que el clima organizacional favorable se asocia a menores niveles de estrés crónico en los trabajadores de un hospital oncológico. ${ }^{7}$ También se ha relacionado el clima laboral con la personalidad, el rendimiento y la satisfacción laboral. ${ }^{8}$

Así pues, la satisfacción laboral puede definirse como una respuesta emocional positiva al puesto y que resulta de la evaluación de si el puesto cumple o permite cumplir los valores laborales del individuo. ${ }^{9}$ También hay estudios que señalan que la satisfacción laboral es un componente de la felicidad ${ }^{10}$, sin embargo, lo que nos satisface no nos hace necesariamente felices. ${ }^{11}$

Algunos de nuestros estudios han relacionado la felicidad con la espiritualidad en el trabajo de forma positiva y muy significativa $^{12}$ y negativamente con el estrés crónico. ${ }^{13}$ En esta misma línea de investigación, el estudio de Sanín y Salanova encontró que la satisfacción laboral se relaciona con el cumplimiento de las normas y el desempeño del trabajador. Asimismo, la flexibilidad y la apertura al cambio -como dimensiones del crecimiento psicológico-, se relacionaron con la satisfacción laboral. ${ }^{14}$

En ese sentido, así como el crecimiento psicológico, otra variable que podria explicar porqué los trabajadores más satisfechos tienen mejor desempeño, es que las personas satisfechas con su trabajo se encuentran bien ajustadas a sus puestos y sus empresas. ${ }^{15}$ Otras investigaciones indican que el liderazgo democrático tiene efectos positivos en la satisfacción laboral, al igual que la cohesión de los trabajadores. ${ }^{16}$

Así pues, mientras en el clima organizacional es posible distinguir entre los factores objetivos y subjetivos, en la satisfacción laboral se suele diferenciar entre los factores intrínsecos y los extrínsecos. Los primeros dependen de la persona, sus aspiraciones, intereses, motivaciones, etc. Los factores extrínsecos, en cambio, tienen que ver con cuestiones fuera de la persona como el reconocimiento, el salario, los beneficios sociales, las condiciones de trabajo, etc. Es importante resaltar que esta postura teórica con respecto a la satisfacción laboral es compatible con las teorías motivacionales y abarca tanto conceptos de la teoría de David McClelland, como de las teorías de Clayton Alderfer, Victor Vroom, Edwin Locke, Richard Hackman y Gray Oldham, cuyos alcances teóricos han sido revisados por diversos autores. ${ }^{17}$

En el presente estudio se pretende analizar las relaciones entre el clima organizacional y la satisfacción laboral en una pequeña empresa de la ciudad de Arequipa, bajo la premisa de que entre ambas variables existen relaciones positivas y significativas. Asimismo, se analizarán las dimensiones de la satisfacción laboral y del clima organizacional en función del sexo de los trabajadores, su tipo de formación profesional y el área de trabajo en la que se ubican. La relación entre estas variables ha sido estudiada desde hace varios años, como en el clásico estudio de Benjamin Schneider y Robert Snyder, quienes reportaron en 1975 que el clima y la satisfacción laboral se relacionaban, según el puesto de la persona. Más recientemente, se han llevado a cabo estudios que retoman la relación entre ambos constructos en diversos contextos laborales y de países con culturas igualmente variadas, confirmándose que el clima organizacional se relaciona o tiene un impacto moderado y significativo en la satisfacción de los trabajadores. ${ }^{19,20,21}$ En nuestro medio, no existen estudios que hayan tocado este tema; por ello el presente trabajo, que se concentra en una empresa privada, constituye un pequeño aporte que se viene a sumar a una línea de investigación que venimos desarrollando en la ciudad de Arequipa (en Perú) sobre el clima organizacional.
MATERIAL Y MÉTODOS

Se trabajó con un diseño de investigación correlacional para valorar las relaciones entre el clima organizacional y la satisfacción laboral.

\section{Muestra}

La muestra está compuesta por 45 trabajadores, 25 varones $(55,6 \%)$ y 20 mujeres $(44,4 \%)$ de 18 a 47 años de edad, con una edad promedio de 35,11 y una desviación estándar de $\pm 12,02$. Asimismo, 31,1\% tiene formación técnica superior y 68,9\% tiene formación universitaria. El 31,1\% trabaja en el área administrativa, 17,8\% en el área de ventas y 51,1\% en el área de operaciones.

\section{Instrumentos}

El Perfil Organizacional de Likert (POL) consta de 25 preguntas con cinco alternativas de respuesta (siempre, casi siempre, a veces, casi nunca, nunca) que se puntúan de 5 a 1 y que ofrecen un puntaje total y puntajes parciales en función de seis dimensiones. La Flexibilidad valora la percepción que tienen los empleados con respecto a las limitaciones en el lugar de trabajo, es decir, el grado en el cual sienten que no hay reglas, procedimientos, políticas y prácticas innecesarias que interfieren con el logro de las tareas y que las nuevas ideas sean fácilmente aceptadas.

Responsabilidad es la percepción que tienen los empleados de tener autoridad delegada, es decir, valora el grado de libertad que tienen los trabajadores para ejecutar sus tareas y actividades asignadas por sus superiores, tomando la responsabilidad de los resultados obtenidos.

La dimensión de Estándares valora la percepción que tienen los empleados sobre el énfasis que pone la gerencia en el mejoramiento del desempeño, incluyendo el grado en el cual la gente siente que se fijan metas retadoras pero realizables. En cuanto a la dimensión Reconocimiento, se refiere al grado en que los empleados perciben que están siendo reconocidos y premiados por realizar un buen trabajo y que dicho reconocimiento esté directa y diferencialmente relacionado con los niveles de desempeño.

Claridad es la percepción de que los trabajadores saben lo que se espera de ellos y que entienden cómo dichas expectativas se relacionan con las metas y objetivos más globales de la organización. Finalmente, Espiritu de Equipo refiere la percepción de los trabajadores del prestigio de la empresa, valora el orgullo que tienen las personas respecto de la organización en que laboran. Indica el grado de confianza de que todos están trabajando hacia un objetivo común.

Este instrumento obedece a un concepto del clima organizacional como percepción del medio interno de la empresa, vinculándolo a su vez con otras variables organizacionales. Además, esta prueba ofrece seis puntajes independientes con respecto a las seis dimensiones del clima organizacional anteriormente señaladas.

Para la realización de este estudio se determinó la validez de este instrumento mediante el método de correlación ítem test, encontrándose que solamente el item 1 y el 3 presentan correlaciones inferiores a 0,20. Los demás items muestran correlaciones moderadas y altas entre 0,24 y 0,78. Se confirmó la estructura de la prueba mediante el análisis factorial que obtuvo un valor KMO de 0,604 y un valor $\mathrm{x}^{2}$ de 764,586 con un nivel de significancia de 0,000 mediante la prueba de esfericidad de Bartlet. Aunque se hallaron siete factores y no seis, estos explican el 75,15\% de la varianza total de la prueba. 
De acuerdo con la rotación varimax, el primer factor se compone de los ítems 2, 6, 11, 13, 15, 20 y 23. El factor dos se compone de los ítems 4, 10, 12 y 17. El factor tres agrupa los items 7, 16 y 21. El cuarto factor agrupa los ítems 5, 24 y 25. El factor cinco abarca los ítems 18 y 19. El sexto factor abarca los ítems 14 y 22. Finalmente, los ítems que saturan en el séptimo factor son el 1 y el 3. Se calculó también la confiabilidad mediante el método de consistencia interna con la prueba alfa de Cronbach y se obtuvo un valor de $\alpha=0,738$. Para valorar la satisfacción laboral se usó la Escala de Satisfacción en el Trabajo de Warr, Cook y Wall, que consta de 15 ítems y dos dimensiones: satisfacción laboral intrínseca y satisfacción laboral extrínseca. Todas las correlaciones item-test resultaron altamente significativas $(\mathrm{p}<0,00)$, y se registraron dentro de un rango de 0,468 y 0,834 . Estos resultados indican que la prueba posee un alto nivel de homogeneidad y, por lo tanto, los reactivos tienen validez de criterio. Para el cálculo de la validez de constructo se obtuvo un valor KMO de 0,773 y la prueba de esfericidad de Bartlet arrojó un valor $\mathrm{x} 2$ de 510,773 significativo $(\mathrm{p}<0,00)$ lo cual sugiere que es posible realizar un análisis factorial.

El análisis de varianza revela que existen tres factores, los cuales explican el 70,367\% de la varianza de la prueba. Al hacer la rotación varimax se obtuvieron tres. Asimismo, se realizó el cálculo de la confiabilidad por medio del método de consistencia interna con la prueba alfa de Cronbach. El índice obtenido es de $\alpha=0,763$. Estos valores indican que la Escala de Satisfacción en el Trabajo es válida y confiable.

\section{Procedimiento}

Para la evaluación de los trabajadores se contó con la autorización de la gerencia siempre que no se mencione el nombre de la empresa. Se aplicaron las pruebas de clima organizacional y satisfacción laboral en las instalaciones de la empresa de forma individual en el horario de trabajo. El tiempo promedio que tomó la aplicación de las pruebas fue de 20 minutos en total.

\section{RESULTADOS}

Para la valoración de los resultados se aplicaron métodos estadísticos descriptivos y de estadística inferencial. Con respecto a los resultados de la evaluación de las variables de estudio, en la Tabla 1 se pueden ver los valores descriptivos del clima organizacional y la satisfacción laboral con sus respectivas dimensiones y factores. Además, se realizó un análisis correlacional de los valores de cada una de las variables y sus respectivas dimensiones. En la Tabla 2 se puede ver que existen relaciones significativas entre el grado de instrucción y la flexibilidad, responsabilidad, reconocimiento, claridad, clima organizacional, satisfacción extrínseca, intrínseca y global. Ni la edad ni el tiempo de servicio se relaciona con las variables de estudio, no de manera significativa al menos. La flexibilidad se relaciona con la responsabilidad, el clima organizacional, la satisfacción laboral extrínseca, intrínseca y global. La dimensión de responsabilidad, en cambio, se relaciona con estándares, reconocimiento, claridad, espíritu de equipo y clima organizacional. Estándares se relaciona con reconocimiento, claridad, espíritu de equipo y clima organizacional. Reconocimiento se relaciona con claridad, espíritu de equipo, clima organizacional, satisfacción laboral extrínseca y global. Claridad se relaciona con espíritu de equipo y clima organizacional, mientras que espíritu de equipo se relaciona solo con clima organizacional. Tanto la satisfacción laboral extrínseca como la intrínseca se relacionan con la satisfacción laboral global.

Tabla 1.

Valores descriptivos del clima organizacional y la satisfacción laboral.

\begin{tabular}{|c|c|c|c|c|c|c|c|c|c|c|}
\hline & Flex & Resp & Est & Recon & Clar & EspEqu & ClimOrg & SatIntrin & SatExtrin & Sat Lab \\
\hline Media & 15,75 & 13,73 & 10,46 & 10,82 & 14,75 & 18,26 & 83,80 & 33,62 & 30,15 & 63,77 \\
\hline Mediana & 15,00 & 14,00 & 10,00 & 10,00 & 14,00 & 18,00 & 84,00 & 35,00 & 31,00 & 63,00 \\
\hline Moda & 15,00 & 12,00 & 10,00 & 10,00 & 14,00 & 16,00 & 81,00 & 38,00 & 28,00 & 59,00 \\
\hline D. estándar & 2,84 & 3,02 & 2,79 & 2,73 & 2,70 & 3,23 & 12,62 & 9,94 & 7,35 & 16,55 \\
\hline Varianza & 8,09 & 9,15 & 7,80 & 7,46 & 7,32 & 10,47 & 159,43 & 98,83 & 54,08 & 273,99 \\
\hline Asimetria & 0,079 & $-0,531$ & $-0,382$ & 0,006 & 0,091 & 0,372 & $-0,217$ & $-0,782$ & $-0,773$ & $-0,631$ \\
\hline Curtosis & 1,029 & 0,452 & $-0,435$ & $-0,236$ & $-0,859$ & $-0,568$ & $-0,756$ & $-0,113$ & 0,818 & $-0,271$ \\
\hline Rango & 15,00 & 13,00 & 11,00 & 12,00 & 10,00 & 13,00 & 49,00 & 37,00 & 35,00 & 62,00 \\
\hline Mínimo & 9,00 & 7,00 & 4,00 & 5,00 & 10,00 & 12,00 & 58,00 & 11,00 & 7,00 & 26,00 \\
\hline Máximo & 24,00 & 20,00 & 15,00 & 17,00 & 20,00 & 25,00 & 107,00 & 48,00 & 42,00 & 88,00 \\
\hline
\end{tabular}

Tabla 2.

Correlaciones entre clima organizacional, satisfacción laboral, edad, grado de instrucción y tiempo de servicios.

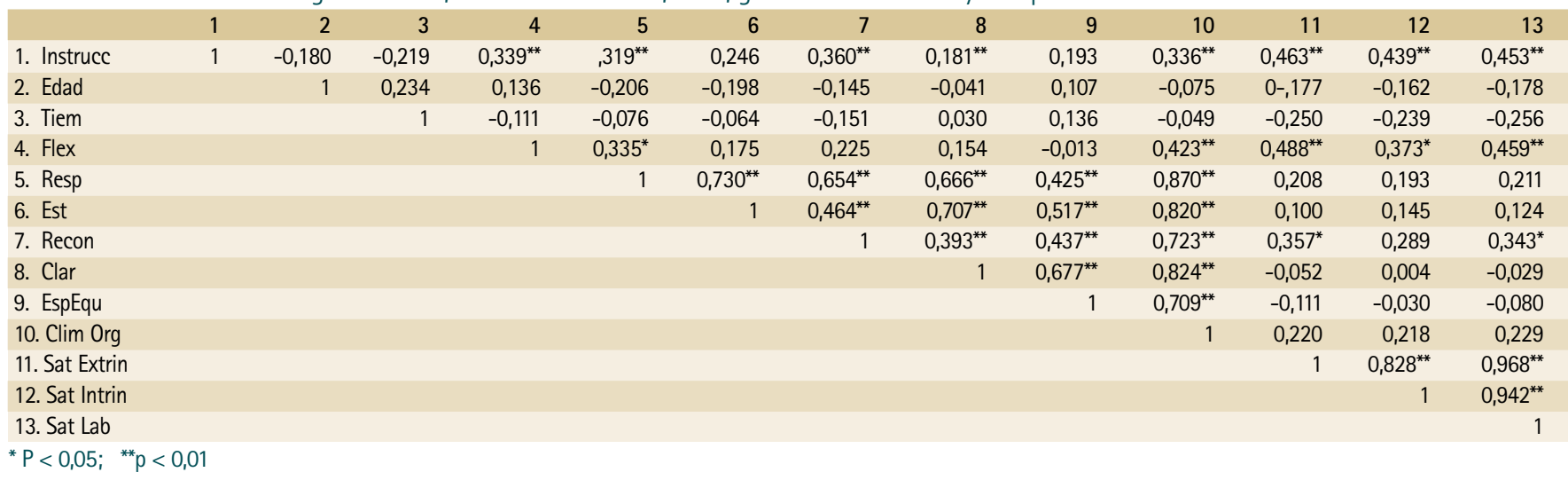


De estos resultados se puede concluir que existe relación entre el clima organizacional y la satisfacción laboral, pero las relaciones entre algunas dimensiones del clima laboral como flexibilidad y reconocimiento son más significativas.

La primera con la satisfacción laboral y sus factores extrínsecos e intrínsecos, y la segunda con la satisfacción laboral extrínseca y la satisfacción laboral global, lo cual es consistente con el hecho de que la satisfacción laboral extrínseca está sujeta a cuestiones externas del sujeto como los elogios de los jefes, los premios recibidos, los bonos, el afecto de los compañeros de trabajo, etc.

Con la finalidad de valorar las diferencias del clima organizacional y de la satisfacción laboral en función del sexo de los trabajadores, dado que la cantidad de trabajadores varones y mujeres es bastante pareja, se realizó una prueba t student. En la Tabla 3 se aprecian las medias de las variables y sus respectivas dimensiones, los valores t y el nivel de significancia de las comparaciones.

Como se puede observar, solo en reconocimiento, espíritu de equipo y satisfacción laboral intrínseca las diferencias entre varones y mujeres son significativas, de modo que son las mujeres quienes se sienten más reconocidas, colaboran mejor entre sí y poseen una motivación extrínseca superior a la de los varones.

Tabla 3.

Medias del CO y la satisfacción laboral de los trabajadores por sexo.

\begin{tabular}{|c|c|c|c|c|c|}
\hline Sexo & & Media & Desviación est. & $\mathrm{t}$ & $p$ \\
\hline \multirow{2}{*}{ Flexibilidad } & Varón & 15,800 & 2,901 & \multirow{2}{*}{0,116} & \multirow{2}{*}{0,908} \\
\hline & Mujer & 15,700 & 2,848 & & \\
\hline \multirow{2}{*}{ Responsabilidad } & Varón & 13,080 & 3,121 & \multirow{2}{*}{$-1,651$} & \multirow{2}{*}{0,106} \\
\hline & Mujer & 14,550 & 2,762 & & \\
\hline \multirow{2}{*}{ Estándares } & Varón & 10,040 & 2,745 & \multirow{2}{*}{$-1,150$} & \multirow{2}{*}{0,257} \\
\hline & Mujer & 11,000 & 2,828 & & \\
\hline \multirow{2}{*}{ Reconocimiento } & Varón & 10,040 & 2,730 & \multirow{2}{*}{$-2,243$} & \multirow{2}{*}{0,030} \\
\hline & Mujer & 11,800 & 2,462 & & \\
\hline \multirow{2}{*}{ Claridad } & Varón & 14,240 & 2,026 & \multirow{2}{*}{$-1,446$} & \multirow{2}{*}{0,155} \\
\hline & Mujer & 15,400 & 3,315 & & \\
\hline \multirow{2}{*}{ Espíritu de equipo } & Varón & 17,200 & 2,483 & \multirow{2}{*}{$-2,634$} & \multirow{2}{*}{0,012} \\
\hline & Mujer & 19,600 & 3,618 & & \\
\hline \multirow{2}{*}{ Clima organizacional } & Varón & 80,400 & 12,315 & \multirow{2}{*}{$-2,096$} & \multirow{2}{*}{0,042} \\
\hline & Mujer & 88,050 & 11,975 & & \\
\hline \multirow{2}{*}{ Satisfacción intrínseca } & Varón & 32,760 & 9,683 & \multirow{2}{*}{$-0,646$} & \multirow{2}{*}{0,522} \\
\hline & Mujer & 34,700 & 10,402 & & \\
\hline \multirow{2}{*}{ Satisfacción extrinseca } & Varón & 27,800 & 7,604 & \multirow{2}{*}{$-2,548$} & \multirow{2}{*}{0,015} \\
\hline & Mujer & 33,100 & 5,981 & & \\
\hline \multirow[t]{2}{*}{ Satisfacción laboral } & Varón & 60,560 & 16,517 & \multirow{2}{*}{$-1,477$} & \multirow{2}{*}{0,147} \\
\hline & Mujer & 67,800 & 16,100 & & \\
\hline
\end{tabular}

Fuente: Elaboración propia.

Se realizó el mismo procedimiento para comparar a los trabajadores con respecto a su grado de instrucción, considerando que aunque todos tienen formación superior, existen trabajadores con formación técnica y otros con formación universitaria. A diferencia de la variable sexo en que la muestra era pareja, en este caso, la mayoría de trabajadores tiene formación universitaria $(68,9 \%)$ y la minoría $(31,1 \%)$ tiene formación técnica. Los resultados se aprecian en la Tabla 4, donde vemos que las diferencias según la formación superior, sea técnica o universitaria, son significativas en las dimensiones de flexibilidad, responsabilidad, estándares y reconocimiento del clima organizacional incluyendo los valores globales de esta variable. También se encontraron diferencias estadísticamente significativas en satisfacción laboral extrínseca, satisfacción laboral intrínseca y satisfacción laboral global; a favor de los trabajadores que cuentan con estudios universitarios de formación superior.
Tabla 4.

Medias del CO y la satisfacción laboral por tipo de instrucción.

\begin{tabular}{|c|c|c|c|c|c|}
\hline Instrucción & & Media & $\begin{array}{l}\text { Desviación } \\
\text { estándar }\end{array}$ & $\mathrm{t}$ & $p$ \\
\hline \multirow{2}{*}{ Flexibilidad } & Técnico & 14,000 & 2,745 & \multirow{2}{*}{$-3,028$} & \multirow{2}{*}{0,004} \\
\hline & Univers. & 16,548 & 2,553 & & \\
\hline \multirow{2}{*}{ Responsabilidad } & Técnico & 11,928 & 3,269 & \multirow{2}{*}{$-2,908$} & \multirow{2}{*}{0,006} \\
\hline & Univers. & 14,548 & 2,566 & & \\
\hline \multirow{2}{*}{ Estándares } & Técnico & 9,214 & 3,017 & \multirow{2}{*}{$-2,098$} & \multirow{2}{*}{0,042} \\
\hline & Univers. & 11,032 & 2,536 & & \\
\hline \multirow{2}{*}{ Reconocimiento } & Técnico & 9,000 & 2,253 & \multirow{2}{*}{$-3,334$} & \multirow{2}{*}{0,002} \\
\hline & Univers. & 11,645 & 2,550 & & \\
\hline \multirow{2}{*}{ Claridad } & Técnico & 14,071 & 3,149 & \multirow{2}{*}{$-1,143$} & \multirow{2}{*}{0,259} \\
\hline & Univers. & 15,064 & 2,475 & & \\
\hline \multirow{2}{*}{ Espíritu de equipo } & Técnico & 17,571 & 3,936 & \multirow{2}{*}{$-0,968$} & \multirow{2}{*}{0,339} \\
\hline & Univers. & 18,580 & 2,884 & & \\
\hline \multirow{2}{*}{ Clima organiz. } & Técnico & 75,785 & 12,867 & \multirow{2}{*}{$-3,135$} & \multirow{2}{*}{0,003} \\
\hline & Univers. & 87,419 & 10,889 & & \\
\hline \multirow{2}{*}{ Satisfacción Intrinseca } & Técnico & 25,071 & 9,887 & \multirow{2}{*}{$-4,724$} & \multirow{2}{*}{0,000} \\
\hline & Univers. & 37,483 & 7,284 & & \\
\hline \multirow{2}{*}{ Satisfacción extrínseca } & Técnico & 25,357 & 4,732 & \multirow{2}{*}{$-3,244$} & \multirow{2}{*}{0,002} \\
\hline & Univers. & 32,322 & 7,350 & & \\
\hline \multirow{2}{*}{ Satisfacción laboral } & Técnico & 50,428 & 13,992 & \multirow{2}{*}{$-4,297$} & \multirow{2}{*}{0,000} \\
\hline & Univers. & 69,806 & 14,010 & & \\
\hline
\end{tabular}

Fuente: Elaboración propia.

Para comparar las puntuaciones de los trabajadores en clima organizacional y satisfacción laboral en función del área de trabajo, se realizó un análisis de varianza. La prueba de homogeneidad de varianzas es significativa para las dimensiones de claridad, espíritu de equipo y la variable clima organizacional, así como para la dimensión satisfacción intrínseca y la variable satisfacción laboral. Dichos valores se aprecian en la Tabla 5 que se muestra a continuación.

Tabla 5.

Prueba de homogeneidad de varianzas.

$\begin{array}{lrrrc} & \text { Estadístico de Levene } & \text { df1 } & \text { df2 } & \text { Sig. } \\ \text { Flexibilidad } & 1,686 & 2 & 42 & 0,198 \\ \text { Responsabilidad } & 1,405 & 2 & 42 & 0,257 \\ \text { Estándares } & 0,807 & 2 & 42 & 0,453 \\ \text { Reconocimiento } & 0,027 & 2 & 42 & 0,974 \\ \text { Claridad } & 2,448 & 2 & 42 & 0,099 \\ \text { Espiritu de Equipo } & 4,168 & 2 & 42 & 0,022 \\ \text { C0 } & 3,252 & 2 & 42 & 0,049 \\ \text { S. intrinseca } & 5,299 & 2 & 42 & 0,009 \\ \text { S. extrinseca } & 1,878 & 2 & 42 & 0,166 \\ \text { S. laboral } & 3,924 & 2 & 42 & 0,027\end{array}$

El análisis de la homogeneidad varianza mediante la prueba de Levene indica que existen diferencias significativas en las dimensiones del clima organizacional de estándares, reconocimiento, espíritu de equipo, así como en el puntaje global de clima organizacional. Para la variable de satisfacción laboral, tanto la dimensión de satisfacción intrínseca, satisfacción extrínseca y la satisfacción laboral como puntaje global son significativas $(\mathrm{p}<0,05)$.

Finalmente, también se hizo un análisis a posteriori con la prueba de Bonferroni para tener mayor precisión entre los valores de las tres áreas de trabajo comparadas. En ese sentido, la Tabla 6 muestra estos valores y evidencia que en la dimensión de estándares del clima organizacional existen diferencias significativas entre las áreas de ventas y administración, administración y operaciones, operaciones y ventas, y en la dimensión de reconocimiento hubo diferencias entre operaciones, administración y ventas. 
Tabla 6.

Correlaciones múltiples con la prueba de Bonferroni.

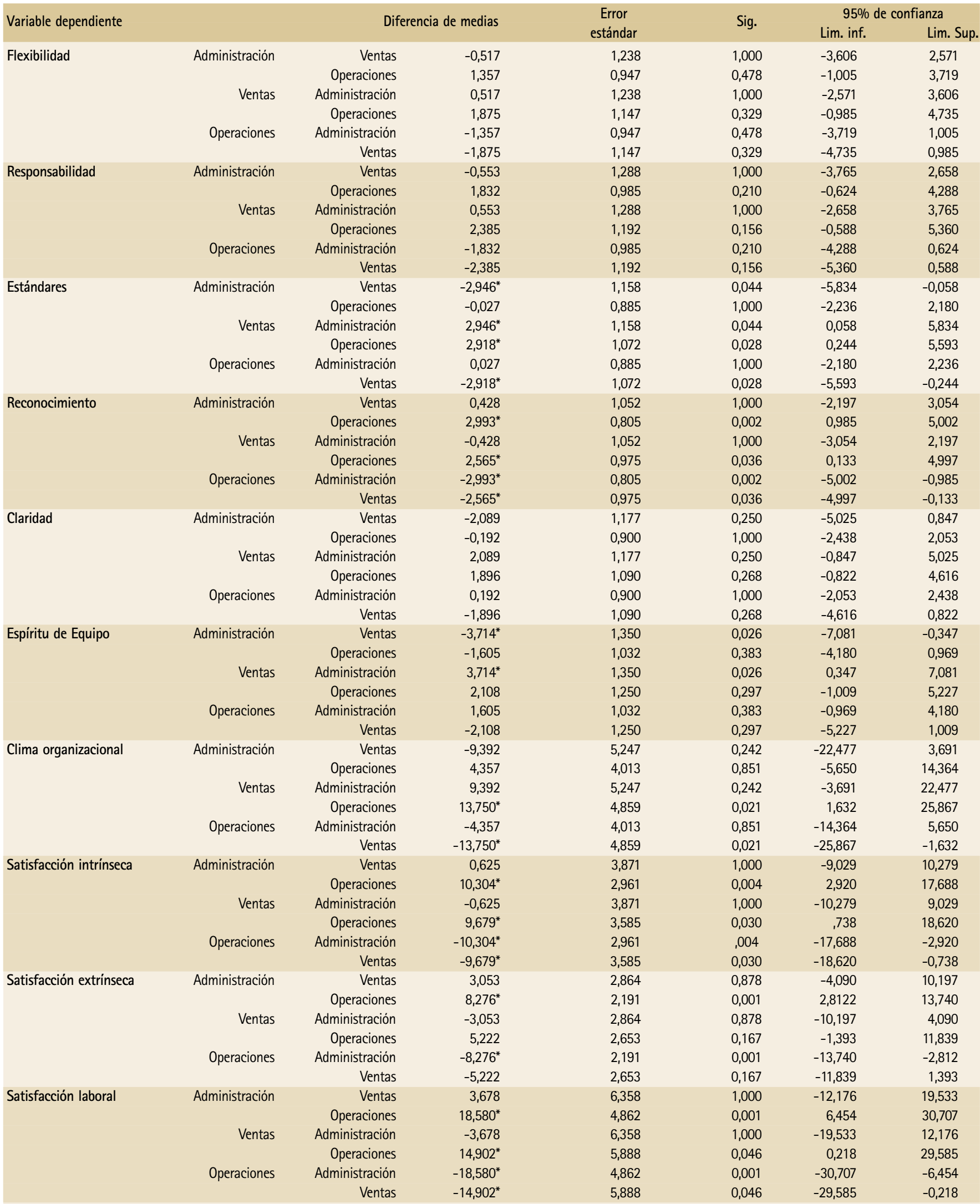


En la dimensión de claridad las diferencias se ubican entre administración y ventas. Igualmente, ocurre con la dimensión espíritu de equipo, donde las diferencias entre administración y ventas son significativas. Con respecto al valor global de clima organizacional, existen diferencias significativas entre las áreas de operaciones y ventas.

En cuanto a la satisfacción laboral intrínseca, las diferencias son significativas entre las áreas de administración y operaciones, así como entre ventas, operaciones y administración. La satisfacción laboral extrínseca reporta diferencias entre las áreas de operaciones y administración. La satisfacción laboral como puntaje global también reporta diferencias significativas entre los trabajadores de las áreas de administración, operaciones y ventas.

Por tanto puede decirse que el área de trabajo sí influye en la percepción del clima organizacional y la satisfacción laboral, en favor de las áreas de administración y ventas por sobre el área de operaciones.

\section{DISCUSIÓN}

Usualmente se define la satisfacción laboral como un estado emocional positivo y placentero de la percepción subjetiva de las experiencias laborales del sujeto ${ }^{22}$, mientras que el clima organizacional se concibe como la percepción que tienen los individuos sobre el ambiente interno de su trabajo.

Aunque no son iguales, el clima organizacional y la satisfacción laboral dependen de las características de la organización y las condiciones de trabajo $\mathrm{y}$, a su vez, ambas son causa de múltiples formas de comportamiento organizacional. Sin embargo, los resultados de algunas investigaciones son contradictorios. Mientras en algunos estudios, el clima organizacional se relaciona con la satisfacción laboral positivamente ${ }^{23}$, los factores de riesgo psicosocial se relacionan negativamente con la satisfacción laboral ${ }^{24}$, y el salario, las relaciones interpersonales, la organización del trabajo y las posibilidades de promoción son fuentes importantes de satisfacción laboral $^{25}$ (como se prevé según la teoría desarrollada en torno a este concepto); otros estudios no parecen confirmar tales hallazgos.

Por ejemplo, en la investigación de Moreira et al. ${ }^{26}$ las condiciones físicas y sanitarias de una muestra de dentistas brasileños no se relacionaron con la satisfacción laboral. De manera similar, en el trabajo de Salgado et al. no se encontró relación entre la satisfacción laboral y el clima organizacional en una pequeña empresa conformada por 17 trabajadores. ${ }^{9}$ Parece ser que la cantidad de trabajadores tiene cierto efecto sobre las variables en mención. De modo que en las empresas grandes las relaciones entre las variables organizacionales tienden a confirmarse, pero en las empresas pequeñas no siempre es así. Posiblemente eso se debe a que en las empresas pequeñas las relaciones interpersonales, también denominadas relaciones humanas, tienden a ser conflictivas, al menos en nuestro contexto local. Un estudio realizado en 159 trabajadores de 18 mypes de uno de los distritos más poblados, se encontró que las relaciones humanas predominantes eran de tipo hostil $^{27}$, lo cual puede tener efectos en la satisfacción laboral y el clima organizacional.

En el presente estudio, a pesar de contar con una muestra pequeña de 45 trabajadores, nuestras hipótesis se han confirmado, ya que el clima organizacional y la satisfacción laboral se han relacionado moderadamente $(r=0,229)$ pero no de manera significativa. En cambio, la flexibilidad, dimensión del clima organizacional que expresa la apertura a la innovación, por un lado, y la rigidez de normas y políticas, por otro, obtuvo un coeficiente de correlación muy significativo $(r=0,459 ; p<0,01)$. Lo cual es consistente con otros estudios que reportan que la flexibilidad es un potente mediador de la satisfacción laboral. ${ }^{14}$ Otra dimensión del clima organizacional que se relaciona con la satisfacción laboral es el reconocimiento, sobre todo con la satisfacción laboral extrinseca $(r=0,357)$ porque el reconocimiento es una fuente de satisfacción fuera de la persona.

$\mathrm{Al}$ respecto, en la ciudad de Arequipa, el reconocimiento es una de las dimensiones del clima organizacional que se ha descrito como deficitaria. ${ }^{2}$ Por ello, no sorprende que se correlacione significativamente con la satisfacción laboral. En ese sentido, es recientemente en los últimos años que las empresas de Arequipa han implementado medidas para reconocer a sus empleados, como elogiar al trabajador del mes o premiar al equipo de trabajo. También se están haciendo reconocimientos públicos y otorgando bonos económicos a los trabajadores. Sin embargo, estas medidas son todavía poco difundidas y se aplican sobre todo en las empresas grandes y medianas.

Con base en estos hallazgos se puede inferir que algunas dimensiones del clima organizacional se relacionan de manera más potente con la satisfacción laboral que el propio clima organizacional. En el estudio de Salgado, Remeseiro e Iglesias ${ }^{9}$ sólo en el factor de relaciones interpersonales se aprecia una correlación significativa entre clima organizacional y satisfacción laboral, por tanto, se concluyó que ambos son constructos relacionados pero independientes entre sí. En nuestro país también se ha reportado que las relaciones interpersonales contribuyen a la satisfacción laboral de los trabajadores. ${ }^{28}$

Para nuestro caso, la satisfacción laboral también se ha relacionado con el grado de instrucción, lo que se refuerza con el análisis comparativo que practicamos con la prueba t student, donde se aprecia que quienes tienen mayor grado de instrucción se sienten más satisfechos laboralmente. Es decir, que los trabajadores con estudios universitarios tienen niveles de satisfacción mayores que quienes tienen estudios técnicos, sobre todo en la satisfacción intrínseca como global. 0 sea que el tener mayor grado de instrucción es fuente de satisfacción personal y actuaría como un motivador intrínseco.

Por otro lado, las mujeres tienen puntuaciones mayores en reconocimiento, espíritu de equipo, clima organizacional y satisfacción extrínseca. Esto se ha reportado en otros trabajos ${ }^{29}$ y se explica porque en el medio laboral latino, donde persiste una idiosincrasia machista, la inserción de las mujeres en el mercado laboral es percibida con mayor relevancia por el género femenino.

Finalmente, los puestos de administración presentan mayor nivel de satisfacción que los trabajadores de ventas y los operarios de acuerdo con el análisis de varianza y la prueba post hoc de Bonferroni. Posiblemente porque las labores de los trabajadores de ventas y operaciones son más demandantes que las de los administrativos; a su vez, las labores de los trabajadores del área de ventas son más demandantes que las de los operarios.

Una manera que se ha sugerido para mejorar la satisfacción laboral ante estas circunstancias es el enriquecimiento del trabajo a través de rotaciones internas, la promoción de una visión integral del trabajo y el empoderamiento, entre otras medidas. Cabe señalar que estas diferencias son mayores en las dimensiones de reconocimiento, estándares y satisfacción laboral; mientras que en flexibilidad, responsabilidad y claridad no. Esto significa que los trabajadores 
sienten en igual medida que hay apertura a la innovación y que conocen sus funciones con claridad; sin embargo, no todos tienen un mismo desempeño, ni se sienten reconocidos en igual medida. Tampoco se sienten satisfechos de la misma forma. Como ya vimos, el grado de instrucción, el sexo y el área de trabajo son factores que explican estas diferencias.

Es sobre la base de estos datos que podrán implementarse estrate- gias de cambio organizacional en esta empresa, lo cual requerirá de una actuación decidida desde los órganos de gobierno. Además, se deberian desarrollar medidas organizacionales e individuales para reducir las diferencias percibidas y generar espacios de apoyo, ya que es probable que, aunque no la hemos evaluado, la salud ocupacional sea deficitaria, sobre todo en las áreas de trabajo y los empleados menos favorecidos por los factores expuestos.
1. Gómez CA. Diseño, construcción y validación de un instrumento que evalúa clima organizacional en empresas colombianas, desde la teoría de respuesta al item. Acta Colom Psicol.2001; 11(4):97-113.

2. Arias WL. Clima organizacional en ocho empresas de Arequipa. Illustro. 2013; 4:39-56.

3. Likert R. El factor humano en la empresa, su dirección y valoración. Bilbao: Deusto; 2002.

4. Chiavenato I. Gestión del talento humano. México: McGraw-Hill; 2009.

5. Moreira JM, Álvarez MC. Clima organizacional en una unidad de riesgo. Emergencias. 2002;14:6-12.

6. León A. Clima organizacional. Antesala del aseguramiento de la calidad. Ingeniería y Desarrollo. 2000; 8:25-32.

7. Arias WL, Zegarra J. Clima organizacional, sindrome de burnout y afrontamiento en trabajadores de un hospital oncológico de Arequipa. RevPsicol (Trujillo). 2013; 15(1):37-54.

8. Guillén $C$, Gala F, Velázquez R. Clima organizacional. En: Guillén $C$, Guill R, coordinadores. Psicologia del trabajo para relaciones laborales. Madrid: McGraw-Hill; 2000. p.164-179.

9. Salgado JF, Remeseiro C, Iglesias M. Clima organizacional y satisfacción laboral en una PYME. Psicothema. 1996;8(2): 329-335.

10. Gamero H. La satisfacción laboral como dimensión de la felicidad. CiencTrab. 2013; 15(47):94-102.

11. Robbins SP, Judge TA. Introducción al comportamiento organizativo. Madrid: Pearson; 2010.

12. Arias WL, Masias MA, Muñoz E, Arpasi M. Espiritualidad en el ambiente laboral y su relación con la felicidad del trabajador. Revlnvestig (Arequipa). 2013; 4:9-33.

13. Arias WL, Masias MA, Justo, 0 . Felicidad, sindrome de burnout y estilos de afrontamiento en una empresa privada. Av. Psicol. 2014; 22(1): de próxima aparición.

14. Sanin JA, Salanova M. Satisfacción laboral: el camino entre el crecimiento psicológico y el desempeño laboral en empresas colombianas industriales y de servicios. UnivPsychol. 2014; 13(1):1-22.

15. Latif $A$, Bashir U. Person organization fit, job satisfaction and turnover intention: an empirical study in the context of PakistanGloAdv Res J Manag Bus Stud. 2013; 2(7):384-388.
16. Pérez PS, Azzolin S. Liderazgo, equipos y grupos de trabajo - su relación con la satisfacción laboral. RevPsicol (Lima). 2013; 31(1):151-169.

17. Schultz DP. Psicología industrial. Bogotá: McGraw-Hill: 1999.

18. Schneider B, Snyder RA. Some relationships between job satisfaction and organization climate. J ApplPsychol. 1975; 60(3):318-328.

19. Castro $M$, Martins $N$. The relationship between organizational climate and employee satisfaction in a South African information and technology organization. J Indust Psychol. 2010; 36(1):1-9.

20. Adeniji AA. Organizational climate as a predictor of employee job satisfaction: evidence from covenant university. Business Intelligence Journal. 2011; 4(1): 151-166

21. Jyoti J. Impact of Organizational Climate on Job Satisfaction, Job Commitment and Intention to Leave: An Empirical Model. Journal of Business Theory and Practice. 2013; 1(1): 66-82.

22. Fonseca Y. Estudio de la satisfacción laboral en los trabajadores de la empresa comercializadora y distribuidora de medicamentos (EMCOMED) de la provincia de Granma. Rev Cuba Salud Trab. 2010; 11(2): 15-19.

23. Arias M. Factores del clima organizacional influyentes en la satisfacción laboral de enfermería, concerniente a los cuidados intensivos neonatales del Hospital Nacional de Niños, 2004. Enfermería en Costa Rica, 2007; 28(1): 12-19.

24. Gómez $P$, Hernández J, Méndez MD. Factores de riesgo psicosocial y satisfacción laboral en una empresa chilena del área de la mineria. CiencTrab. 2014; 16(49): 9-16.

25. Moreira M, Saliba CA, Saliba TA, Salibas O, Saliba SA. Condiciones físicas y sanitarias del trabajo y satisfacción de cirujanos dentistas brasileños con el empleo público. RevCub Salud Trab. 2013; 14(3): 45-54.

26. Arias $W L$, Justo 0 . Satisfacción laboral en trabajadores de dos tiendas por departamento: un estudio comparativo. CiencTrab. 2013; 15(47): 41-46.

27. Arias WL, Jiménez NA. Relaciones humanas en mypes de Arequipa. ContNeg. 2013; 8(16): 48-60.

28. Yañez $R$, Arenas $M$, Ripoll M. El impacto de las relaciones interpersonales en la satisfacción laboral general. Liberabit. 2010; 16(2): 193-202.

29. Gómez A, Benítez C, Guillén c, Gala FJ, Lupiani M. Motivación y satisfacción laboral. En: Guillén $C$, Guil $R$, coordinadores. Psicologia del trabajo para relaciones laborales. Madrid: McGraw-Hill; 2000. p. 195-209. 